\title{
Le laboratoire du praticien est encore rentable en 2004
}

\author{
M. Hug, Président Commission Laboratoire CMPR
}

Correspondance: Dr Markus Hug Rue de la Préfecture 1 CH-2800 Delémont
L'introduction du tarif fédéral de la liste des analyses (LA) au $1^{\text {er }}$ janvier 2004 a provoqué des baisses importantes des tarifs de laboratoire du cabinet du praticien dans certains cantons. Pour d'autres, la LA faisait déjà partie de leur facturation. Cette adaptation a toutefois certainement évité une baisse encore plus massive du tarif (une réduction de la valeur du point LA à 0,80 était demandée à cette époque par l'OFSP). Il n'est pas inutile de rappeler ici que certains tarifs cantonaux avaient obtenu des valeurs doubles de la moyenne suisse (dans une comparaison des paramètres les plus fréquemment utilisés dans l'étude du laboratoire de 2000).

L'étude du laboratoire du praticien des Professeurs Felix Gutzwiller et Thomas Szucs a confirmé l'utilité médicale et économique du laboratoire du praticien. L'économicité appelle un commentaire lié à la rentabilité. La définition de la rentabilité mérite d'être faite en fonction des critères définis non seulement par le corps médical, mais également par les partenaires des assurances sociales et de l'OFSP. Le chiffre habituellement articulé de 25 à 30\% est généralement admis. Il est pour certains proche de la non-rentabilité, mais cette estimation est laissée à la libre appréciation de chacun. Il nous paraît pour notre part raisonnable, et il ne devrait en aucun cas être abaissé; il doit surtout être valable pour les confrères utilisant les examens de laboratoire dans le cadre d'une démarche diagnostique raisonnable et raisonnée. Cette marge doit également être applicable aux confrères effectuant moins de 130 analyses par semaine (moyenne déterminée dans l'étude du laboratoire du praticien).

Il est utile de rappeler que le laboratoire du praticien est soumis à des contrôles de qualité réguliers; les contrôles de qualité externes per- mettent de détecter les erreurs grossières dans les analyses. L'introduction des contrôles de qualité internes chaque 15 jours permettra d'améliorer sensiblement la précision des examens. Cet intervalle paraît raisonnable avec la fiabilité des analyseurs modernes. Rappelons que les grands laboratoires sont tenus d'effectuer au moins deux contrôles internes quotidiens et qu'ils dépensent près de $20 \%$ de leur chiffre d'affaires pour les divers contrôles. La tenue du journal du laboratoire et l'exploitation des résultats des contrôles de qualité (en fonction des critères définis dans le document de l'attestation de formation complémentaire du laboratoire du praticien AFCLP) permettront au médecin de disposer de résultats fiables pour ses analyses. Il pourra donc les intégrer dans sa démarche diagnostique et le suivi du patient, en garantissant une excellente qualité de ses prestations de laboratoire.

La problématique du laboratoire est discutée dans de multiples commissions fédérales, qui se disputent parfois certaines parts de compétences. Il n'en reste pas moins que c'est le Département fédéral de l'intérieur qui a le dernier mot. Nous nous engageons fermement pour le laboratoire du praticien comme outil indispensable à la bonne pratique médicale et des tarifs répondant aux exigences de qualité élevées valables en Suisse. Les résultats du diagnostic de présence entraînent des économies significatives.

Soutenez donc les efforts de la FMH et des confrères engagés dans ce domaine pour le maintien d'un laboratoire du praticien de qualité et ... rentable. 